\title{
OSAHS
}

\section{The association between dysphagia and OSA}

\section{Disfagia e OSA}

\author{
Lorenzo Marra', Giada Cavallaro², Valentina Di Lecce³, Giorgio Castellana4, Carla Santomasi3 ${ }^{3}$ Vincenzo Di Nicola², \\ Nicola Antonio Adolfo Quaranta ${ }^{2}$, Giovanna Elisiana Carpagnano ${ }^{3}$, Onofrio Resta ${ }^{3}$, Maria Luisa Fiorella ${ }^{2}$ \\ ${ }^{1}$ Respiratory Disease Unit, "San Giuseppe Moscati" Hospital, Taranto, Italy; ${ }^{2}$ Otolaryngology Unit, Department of Biomedical \\ Sciences, Neuroscience and Sensory Organs, University of Bari "Aldo Moro", Italy; ${ }^{3}$ Institute of Respiratory Disease, Department of \\ Basic Medical Science, Neuroscience and Sensory Organs, University of Bari "Aldo Moro", Italy; ${ }^{4}$ Respiratory Rehabilitation of the \\ Institute of Bari, Istituti Clinici Scientifici Maugeri IRCCS, Bari, Italy
}

\section{SUMMARY}

Objective. The aim of our study was to investigate the presence of dysphagia in patients with Obstructive Sleep Apnoea (OSA) and to correlate swallowing impairment with hypnologic and anatomic parameters.

Methods. The study population includes 36 patients suffering from OSA. Patients were divided into two groups using the presence of dysphagia as a distinctive parameter. Group 1 included 27 OSA patients without signs of dysphagia and Group 2 included 9 OSA patients with signs of dysphagia.

Results. The age of patients in Group 2 was higher compared with the age of patients in Group 1. Analysis of Continuous Positive Airway Pressure (CPAP), obtained in the titration phase, showed that OSA patients with signs of dysphagia required a higher level of CPAP pressure than those who were not affected by swallowing abnormalities (12.6 $\pm 1 \mathrm{vs}$ $10.5 \pm 1.9 \mathrm{p}=0.003)$. No other differences in anthropometric, hypnologic, or arterial blood gas values were found between the two groups.

Conclusions. In clinical practice, all OSA patients should undergo a complete ENT exam, including assessment of swallowing, before CPAP therapy is started. This may predict the need for higher CPAP pressure settings to resolve apnoea episodes in the presence of dysphagia as well as guide the choice of CPAP interfaces (orofacial vs. nasal) in these patients.

KEY WORDS: obstructive sleep apnoea syndrome, dysphagia, swallowing impairment, CPAP

\section{RIASSUNTO}

Obiettivo. Il nostro studio ha avuto come obiettivo la ricerca nei pazienti OSA di evidenze di disfagia e l'associazione della presenza di disfagia alla anatomia delle prime vie aerodigestive e ai parametri ipnologici ed emogasanalitici di questi pazienti.

Metodi. Tutti i pazienti sono stati sottoposti a emogasanalisi, spirometria, rinomanometria, videofibrorinolaringoscopia, valutazione fibroendoscopica della deglutizione (FEES), polisonnografia notturna, studio di titolazione della pressione di lavoro di CPAP, somministrazione del questionario "SWAL-QOL".

Risultati. Il $25 \%$ della popolazione studiata ha presentato segni subclinici di disfagia. Il gruppo dei pazienti non disfagici era formato da soggetti più giovani rispetto al gruppo dei pazienti disfagici. Il distretto orofaringeo è risultato essere il sito di ostruzione più frequente per entrambi i gruppi. Nello studio di titolazione della pressione di lavoro di CPAP, i pazienti disfagici necessitano di valori di pressione più alti.

Conclusioni. Un paziente OSA su 4 ha presentato segni di disfagia. La disfagia è associata a valori più elevati di pressione allo studio di titolazione della CPAP. I pazienti OSA hanno un rischio più elevato di presentare disturbi di deglutizione con l'avanzare dell'età rispetto alla popolazione generale.

PAROLE CHIAVE: sindrome delle apnee ostruttive nel sonno, disfagia, disturbi di deglutizione, CPAP
Received: March 22, 2021

Accepted: September 29, 2021

Published online: February 7, 2022

Correspondence

Giada Cavallaro

Otolaryngology Unit, Department of Biomedical Sciences, Neuroscience and Sensory Organs, University of Bari "Aldo Moro",

p.zza G. Cesare, 70120 Bari, Italy

E-mail: giadacavallaro@live.it

How to cite this article: Marra L, Cavallaro G, Di Lecce V, et al. The association between dysphagia and OSA. Acta Otorhinolaryngol Ital 2022;42:82-88. https://doi. org/10.14639/0392-100X-N1578

(C) Società Italiana di Otorinolaringoiatria e Chirurgia Cervico-Facciale

\section{(c) (i) $(9)$}

This is an open access article distributed in accordance with the CC-BY-NC-ND (Creative Commons Attribution-NonCommercial-NoDerivatives 4.0 International) license. The article can be used by giving appropriate credit and mentioning the license, but only for non-commercial purposes and only in the original version. For further information: https:// creativecommons.org/licenses/by-nc-nd/4.0/deed.en 


\section{Introduction}

Obstructive Sleep Apnoea (OSA) is characterised by repeated episodes of upper airway occlusion, associated with oxyhaemoglobin desaturations and brief awakenings from sleep (arousals) ${ }^{1}$. Due to sleep fragmentation, signs and symptoms of OSA include snoring, abrupt awakenings accompanied by gasping or choking and excessive daytime sleepiness. This latter condition inevitably leads to an increase in cardiovascular risk (risk of arterial hypertension, myocardial infarction and cerebral ischaemia) ${ }^{2}$ and worsening of the subject's quality of life, as well as an obvious increase in the frequency of road or occupational accidents ${ }^{3}$ The disruption of the oro-nasal airflow lasts, by definition, for at least 10 seconds and can be complete (apnoea) or partial (hypopnoea) ${ }^{4}$.

The severity of OSA is scored according to the number of apnoeas or hypopnoeas, recorded during the study, per hour of sleep (apnoea/hypopnoea index - AHI). OSA is therefore classified as mild (AHI $>5$, but $<15$ per hour), moderate (AHI $>15$, but $<30$ per hour) and severe $\left(\mathrm{AHI}>30\right.$ per hour ${ }^{5}$. During nocturnal respiratory events, the size of the upper airway depends on the balance between forces that would collapse the airway (such as negative intraluminal pressure and increased tissue-extraluminal pressure) and those that maintain airway (contraction of pharyngeal dilator muscles). The most frequent sites of pharyngeal collapse are the soft palate, lateral pharyngeal walls, palatine tonsils and base of the tongue. The larynx can also be involved as a site of obstruction.

During sleep, the compensatory tonic input to the motor neurons of the pharyngeal dilator muscle is lost, causing collapse of the pharyngeal airway until chemoreceptor stimuli activate the neuromuscular compensatory functions that maintain airway patency. The use of CPAP or surgical treatments of OSA reduce collapse in the upper airway tract between the supraglottic region of the larynx and hypopharynx, which results in improved oxyhaemoglobin saturation and reduced hypercapnia ${ }^{6}$.

The risk factors for OSA include anatomic-functional abnormalities in the nasal and nasopharyngeal area ("nasopharyngeal level"), in the back palatal area and at the Isthmus of fauces ("oropharyngeal level") and in the region that includes the hyoglossus-mandibular complex and the rear lingual space ("hypopharyngeal level") ${ }^{7}$.

Specifically, Waldeyer ring hypertrophy is widespread in the OSA paediatric population ${ }^{8}$, while in adulthood, both the alterations of the splanchnocranial skeletal morphotype and the anatomical pathologies and dysmorphisms of the upper aero-digestive tract have been reported to play a decisive role in the pathogenesis of OSA (nasal growths, nasal septum deviation, choanal atresia, macroglossia, hypertrophy of the soft palate, uvula, lingual tonsil and turbinates, malformations of epiglottis, fat deposits around the pharynx) ${ }^{9}$.

The complications of OSA may include cognitive impairment, depression, nocturnal oesophageal reflux and nocturia ${ }^{10}$.

Some studies have shown that patients with OSA may be affected by swallowing dysfunctions, which reflects the abnormal function of nerves and muscles in the suprapharynx ${ }^{11}$.

The aim of the present study was to investigate the presence of oropharyngeal dysphagia in patients with OSA and to correlate swallowing impairment with hypnologic, anatomic and arterial blood gas values.

\section{Materials and methods}

Thirty-six OSA patients from the Sleep Disorder Centre of "Department of Respiratory Disease" were recruited and assessed at "ENT Clinic", University of Bari. Of the 36 patients enrolled, 26 were males and 10 females (respectively, $72.2 \%$ and $27.8 \%$ ).

The exclusion criteria included age $>75$ years and $<45$ years, daytime respiratory failure, Overlap Syndrome (Chronic Obstructive Pulmonary Disease and OSA), obesity hypoventilation syndrome (OHS), neuromuscular disorders, neurological diseases, gastroenterological diseases, craniofacial malformations, decompensated clinical and psychiatric illnesses and patients who had used CPAP therapy in the preceding three months.

Participants were approached and informed about the study objectives and significance, before confirming their availability to take part in the present research.

All patients underwent the following tests:

- recent and remote pathological anamnesis, family history and pharmacological anamnesis. Patients were also interrogated about dietary and other habits, comorbidities and general symptoms of sleep disorders.

- Body Mass Index (BMI);

- Epworth Sleepiness Scale (ESS);

- arterial blood gas analysis;

- spirometry;

- full-night cardiorespiratory polygraphy recording (NoxT3, Nox Medical Inc. Reykjavik, Iceland). The following data were collected: snoring sound, body position, activity, heart rate, nasal pressure, oxyhaemoglobin saturation, and thoracic and abdominal movements. The hypnological parameters obtained were evaluated by a physician experienced in sleep breathing disorders;

- clinical examination to assess the anatomical characteristics of the upper airways [Friedman tongue posi- 
tion staging system (FTP), tonsil hypertrophy grading scale] ${ }^{12}$;

- rhinomanometry;

- fiber optic nasopharyngolaryngoscopy of the upper airways, during which a modified Muller maneuver at oropharyngeal and hypopharyngeal levels was carried out according to the nose oropharynx hypopharynx and larynx (NOHL) classification ${ }^{13}$;

- fibre optic endoscopic evaluation of swallowing (FEES) using boluses of different texture: liquid, semisolid and solid bolus. We analysed the anatomical structures and the functional aspects of the swallowing events, according to the following criteria : (a) premature oral leakage, if the bolus entered the pharynx without eliciting the swallowing reflex; (b) laryngeal penetration if the bolus penetrated the laryngeal vestibule but not below the vocal cords; (c) tracheal aspiration if the bolus passed below the vocal cords; lastly, (d) pharyngeal stasis if the bolus remained in the valleculae and/or pyriform sinuses after the swallowing sequence, using the Yale Pharyngeal Residue Severity Rating Scale ${ }^{14}$. The Yale Pharyngeal Residue Severity Rating Scale is a standardised, anatomically defined, image-based assessment of postswallow pharyngeal residue, based on a 5-grade rating of the amount of residue in two locations, the vallecula and the pyriform sinus as observed during fiberoptic endoscopic evaluation of swallowing;

- the Italian version of SWAL-QOL questionnaire on quality of life in dysphagia ${ }^{15}$;

- CPAP titration study.

The results are presented as percentages for categorical variables and as mean \pm standard deviation (SD) for continuous variables. The proportions were compared using the Pearson $\chi^{2}$ test. The means were compared using the two-tailed Student's t-test. A p value $<0.05$ was considered as significant.

\section{Results}

The clinical and demographic characteristics of the 36 patients in this study are presented in Table I.

Oropharyngeal examination revealed that $53 \%$ of subjects $(\mathrm{n}=19)$ had grade $1,31 \%$ had grade II $(\mathrm{n}=11)$ and 16 $(n=6)$ had grade III tonsil size.

According to the Friedman tongue position staging system, $11 \%$ of the cases $(n=4)$ were classified as FTP stage 1 , $17 \%(\mathrm{n}=6)$ as stage $2,39 \%(\mathrm{n}=14)$ as stage 3 and $33 \%$ $(\mathrm{n}=12)$ as stage 4 .

The results of FEES demonstrated that a post-swallow residue with solid bolus texture was present in $25 \%$ of patients $(\mathrm{n}=9)$. According to the Yale Pharyngeal Residue Sever-
Table I. Demographic and clinical characteristics of the cohort.

\begin{tabular}{lc} 
Patients $(\mathrm{n})$ & 36 \\
Age (years, Mean \pm SD) & $60.7 \pm 11.1$ \\
Male gender n (\%) & $26(72.2 \%)$ \\
BMI (Mean \pm SD) & $33.6 \pm 6.9$ \\
ESS (Mean \pm SD) & $7 \pm 5$ \\
OSA severity n (\%) & \\
Mild & $3(8 \%)$ \\
Moderate & $6(17 \%)$ \\
Severe & $27(75 \%)$ \\
CPAP Pressure (Mean \pm SD) & $11.1 \pm 1.9$ \\
Tonsil volume n (\%) & \\
Grade 1 & $19(53 \%)$ \\
Grade 2 & $11(31 \%)$ \\
Grade 3 & $6(16 \%)$ \\
Friedman tongue position staging system n (\%) & \\
Grade 1 & $4(11 \%)$ \\
Grade 2 & $6(17 \%)$ \\
Grade 3 & $14(39 \%)$ \\
Grade 4 & $12(33 \%)$ \\
Post-swallowing stasis at FEES n (\%) & $9(25 \%)$ \\
\hline SD: &
\end{tabular}

SD: standard deviation; BMI: Body Mass Index; ESS: Epworth Sleepiness Scale; OSA: Obstructive Sleep Apnoea Syndrome; CPAP: continuous positive airway pressure; FEES: Fibre-optic endoscopic evaluation of swallowing.

ity Rating Scale, none/trace grade of severity of vallecular residue was observed in 2 subjects $(22.2 \%)$ mild grade of severity of vallecular residue was observed in $3(33.3 \%)$ and moderate grade of severity in $4(44.4 \%)$ subjects, but no participant showed severe vallecular residue.

As for the pyriform sinus location, 3 (33.3\%) respondents showed none/trace, and 4 patients $(44.4 \%)$ had mild severity of residue. While the remaining 2 (22.2\%) subjects had moderate severity of residue, no individuals with severe residue in the pyriform sinuses were observed.

In the group of patients who presented bolus residue after swallowing, 8 patients had severe OSA (88.9\%) and 1 patient had moderate OSA (11.1\%).

No cases of laryngeal penetration or tracheal aspiration were observed. We used the NOHL classification system and found that the oropharyngeal level was the most frequent obstruction site (average value $2.5 \pm 1.13$ ) followed by the hypopharynx level (average value $=1.62 \pm 1.04$ ), the larynx (average value $0.26 \pm 0.57$ ) and nasal level (average value $0.18 \pm 0.39$ ) (Fig. 1).

Using the presence of dysphagia as a distinctive parameter, the cohort of patients was divided into two groups. Group 1 included 27 patients with OSA without signs of dysphagia and Group 2 included 9 patients with diagnosis of OSA and signs of dysphagia. 


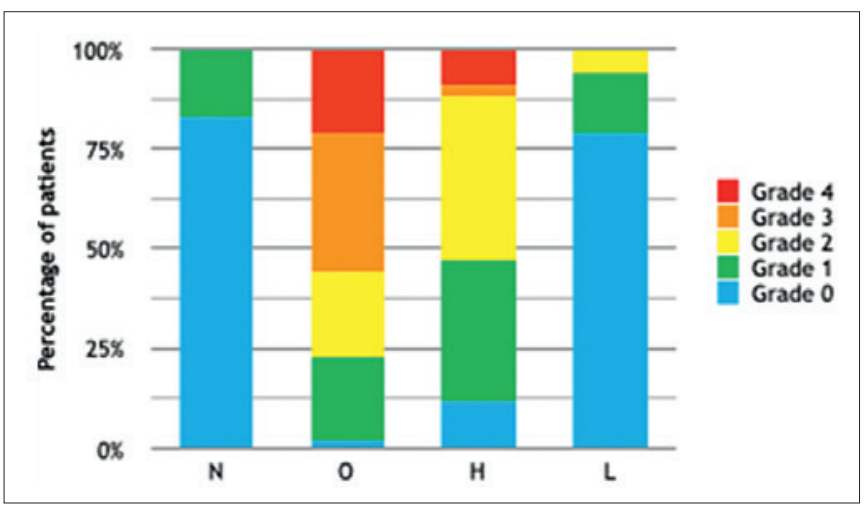

Figure 1. NOHL classification. N: Nose; O: oropharynx; H: hypopharynx; $L$ : larynx; percentage of patients and grade of obstruction.

Anthropometric, hypnologic and arterial blood gas values were compared between the two groups: Patients in Group 2 (dysphagia) were older than patients in Group 1 (no dysphagia). Statistically significant differences were not detected in other items (Tab. II).

No significant differences were found between the two groups with respect to the grading of tonsil size, Friedman tongue position staging system, sites of upper airway collapse, or nasal resistances (Tab. III).

There was also no significant difference between the SWAL-QOL scores of patients with and without signs of dysphagia (Tab. IV).

Finally, we analysed the CPAP pressures obtained in the titration phase in the 2 groups: OSA patients with signs of dysphagia required higher level of CPAP pressure than OSA patients not affected by swallowing abnormalities $(p=0.003)($ Tab. V).

\section{Discussion}

Although our sample size is not large, the OSA patients enrolled (average age 60.7 years) showed a prevalence of dysphagia of $25 \%$. The recent literature ${ }^{16}$ has already highlighted a prevalence of swallowing disorders between $16 \%$ and $78 \%$ in the population affected by OSA under the age of 75 years, which is consistent with our result. Furthermore, our data show that the perception of swallowing abnormalities among the same type of patients appears to have little-to-no significance.

Our patients completed autonomously the Italian version of the SWAL-QOL test before FEES examination: the SWAL-QOL scores of patients with and without signs of dysphagia (Group 1 and Group 2, respectively) showed no significant difference between the two groups, suggesting that patients who suffer from OSA may present subclinical manifestations of abnormal swallowing. Schindler et al.
Table II. Anthropometric, hypnologic and arterial blood gas values.

$\begin{array}{lccc} & \begin{array}{c}\text { Group 1 } \\ \text { (n. 27) }\end{array} & \begin{array}{c}\text { Group 2 } \\ \text { (n. 9) }\end{array} & \text { p } \\ \text { Age (years) } & 58.3 \pm 10.5 & 67.9 \pm 10 & 0.02 \\ \text { BMl } & 33.6 \pm 7.4 & 33.7 \pm 5 & \text { Ns } \\ \text { Gender (M) n(\%) } & 18.0(66.6) & 8.0(88.8 \%) & \text { Ns } \\ \text { AHI } & 38.1 \pm 22.6 & 44.8 \pm 17 & \text { Ns } \\ \text { ODI } & 35.6 \pm 23.1 & 42.5 \pm 19.5 & \text { Ns } \\ \text { TST90 } & 26.9 \pm 26.7 & 30.6 \pm 17.6 & \text { Ns } \\ \text { Mean nocturnal S02 } & 92.0 \pm 2.3 & 91.3 \pm 1.8 & \text { Ns } \\ \text { p02 } & 83.4 \pm 15.2 & 77.4 \pm 11.1 & \text { Ns } \\ \text { pC02 } & 40.6 \pm 3.9 & 40.1 \pm 3.5 & \text { Ns } \\ \text { HC03- } & 27.3 \pm 3.2 & 26.9 \pm 2.8 & \text { Ns } \\ \text { Diurnal S02 } & 96.1 \pm 1.9 & 95.3 \pm 1.8 & \text { Ns }\end{array}$

BMI: Body Mass Index; AHI: Apnoea Hypopnoea Index; ODI: Oxygen Desaturation Index; TST90: Total Sleep Time with oxyhaemoglobin saturation below 90\%; S02: oxyhaemoglobin saturation. Ns: Not statistically significant.

Table III. Tonsillar size, Friedman tongue position staging system, upper airway collapsibility and nasal resistances.

\begin{tabular}{lccc} 
& Group 1 & Group 2 & p \\
$\begin{array}{l}\text { Tonsil size } \\
\text { Grade 3-4 }\end{array}$ & 3 & 2 & Ns \\
Grade 1-2 & 24 & 7 & Ns \\
$\begin{array}{l}\text { Friedman tongue position } \\
\text { staging system }\end{array}$ & & & \\
$\quad$ Grade 3-4 & 17 & 8 & Ns \\
Grade 1-2 & 10 & 1 & Ns \\
Upper airway collapsibility & & & \\
$\quad$ Oropharynx & 18 & 6 & Ns \\
Other district & 9 & 3 & Ns \\
Nasal resistances & & & \\
Yes & 9 & 4 & Ns \\
No & 18 & 5 & Ns \\
\hline
\end{tabular}

Group 1: patients with OSA without dysphagia. Group 2: patients with OSA and signs of dysphagia. Ns: Not statistically significant.

enrolled 72 patients (54 men and 18 women); all patients underwent fibre optic evaluation of swallowing using boluses of different textures and each patient also completed the SWAL-QOL questionnaire: 64 patients $(89 \%)$ presented spillage, $20(28 \%)$ presented piecemeal deglutition, 26 patients $(36 \%)$ presented penetration and $30 \%$ presented retention. Comparing the SWAL-QOL scales in OSA patients with and without signs of dysphagia, they found a small difference in only two spheres (general burden and symptoms) of the SWAL-QOL questionnaire. These studies show that the evaluation of dysphagia cannot be performed exclusively using self-assessment questionnaires, but 
Table IV. The SWAL-QOL scores.

\begin{tabular}{lccc} 
& Group 1 & Group 2 & p \\
Burden & $9 \pm 1.6$ & $8.7 \pm 2.3$ & Ns \\
Eating desire & $14.7 \pm 3.8$ & $13.4 \pm 2.2$ & Ns \\
Eating duration & $9.3 \pm 1.3$ & $8.7 \pm 2.1$ & Ns \\
Symptoms & $61 \pm 6.9$ & $59 \pm 8.5$ & Ns \\
Food Selection & $8.4 \pm 2.4$ & $8.3 \pm 2.3$ & Ns \\
Communication & $8.9 \pm 2$ & $9.3 \pm 1.1$ & Ns \\
Fear & $17.7 \pm 3.3$ & $17.6 \pm 3.5$ & Ns \\
Mental health & $22.6 \pm 4.5$ & $22.8 \pm 3.4$ & Ns \\
Social functioning & $22.8 \pm 4$ & $22.9 \pm 2.4$ & Ns \\
Fatigue & $10.4 \pm 3.3$ & $8.4 \pm 4.4$ & Ns \\
Sleep & $7.3 \pm 2.4$ & $6.8 \pm 2.7$ & Ns \\
\hline
\end{tabular}

SWAL-QOL questionnaire dysphagia-related quality of life concepts (food selection, burden, mental health, social functioning, fear, eating duration, eating desire, communication) and SWAL-QOL general quality of life concepts (sleep and fatigue). Group 1: patients with OSA without dysphagia. Group 2: patients with OSA and signs of dysphagia. Ns: Not statistically significant.

Table V. CPAP titration.

\begin{tabular}{lccc} 
& Group 1 & Group 2 & p \\
CPAP titratiomn (cmH20) & $10.5 \pm 1.9$ & $12.6 \pm 1$ & 0.003 \\
\hline
\end{tabular}

CPAP: continuous positive airway pressure. Group 1: patients with OSA without dysphagia. Group 2: patients with OSA and signs of dysphagia.

that performing instrumental investigations is crucial ${ }^{17}$. In particular, 41 heavy snorers and 15 non-snoring volunteers were video-radiographically examined by Jaghagen et al. with regards to the oral and oropharyngeal phases of swallowing. The most common finding of abnormal pharyngeal swallowing function in snorers was repeated premature bolus leakage down to different levels of the pharynx before the swallowing reflex was elicited $(51 \%)$, followed by premature bolus leakage that intermittently continued down to the piriform sinus (12\%) and the presence of bolus residual in pharynx $(10 \%)^{18}$. Valbuza et al. studied 11 patients with OSA ad 14 non-snoring volunteers who were evaluated using FEES: $64 \%$ of OSA patients presented premature oral leakage, $55 \%$ presented pharyngeal stasis of the bolus after swallowing and no cases of laryngeal penetration or tracheal aspiration were observed, which explains why patients with swallowing dysfunction remained asymptomatic ${ }^{19}$. Recently, 60 patients with OSA were subjected to cephalometric evaluation, orofacial myofunctional evaluation with score (OMES-E) and swallow video fluoroscopy exam by Valarelli et al. The OSA patients showed substantial orofacial and pharyngeal muscular impairment, swallowing disorders, narrower pharynx and lower hyoid position compared to a control group ${ }^{20}$.

Our study demonstrated that FEES is particularly reliable for the study of dysphagia and revealed no significant difference between patients with and without dysphagia in the site of obstruction during Muller maneuver. Additionally, no anatomical differences were noted between the two groups in terms of Friedman tongue position staging system, grading of tonsil size and nasal resistances. CPAP titration showed that OSA patients with dysphagia required higher levels of pressure compared to patients without swallowing disorders. This finding could be dependent on the lower genioglossus muscle responsiveness during sleep that can be observed in some OSA patients, according to a study by Eckert et al., where the author analysed the pathophysiologic causes of OSA and proposed a phenotypical definition of disease. Eckert's study shows that more than $30 \%$ of OSA patients seem to have a poor muscle responsiveness, as measured by genioglossus electromyography ${ }^{21}$. The success of therapy in this type of OSA patients with dysphagia should include careful follow-up to ensure a good compliance of the patient, despite the high CPAP pressure setting. This may also help improve the patient acceptance of CPAP therapy.

Moreover, the treatment of OSA with CPAP seems to be able to reverse the endoscopic findings of swallowing dysfunction and to improve the quality of life, according to the criteria measured by the SWAL-QOL questionnaire, as demonstrated in the study by Caparroz et al. on 70 adult patients with moderate or severe OSA. The study analysed the signs and symptoms of oropharyngeal dysphagia using FEES and SWAL-QOL in 18 patients with signs of dysphagia, of whom 12 were treated with CPAP for 3 months. The results revealed that the treatment of OSA with CPAP was able to reverse the endoscopic findings of dysphagia and improve quality of life, as measured by the SWAL-QOL test ${ }^{22}$.

Moreover, assessing the presence of dysphagia in OSA patients plays a fundamental role in prevention of one of the most frequent complications in swallowing deficiency: inhalation pneumonia. The incidence of inhalation pneumonia in the elderly rises with increasing age and Cabre et al. showed that, in a population of 134 patients, aged over 75 years, hospitalised for pneumonia, 55.2\% presented signs of oropharyngeal aspiration. Dysphagia can therefore be considered a poor prognostic factor in terms of mortality ${ }^{23}$ but OSA alone, per se, seems to carry a greater risk of developing pneumonia ${ }^{24}$. Chiner et al. demonstrated that the presence of OSA is associated with a greater risk of developing community acquired pneumonia, and AHI correlates with the severity of infection ${ }^{25}$. This connection between OSA and the pulmonary infections provide strong evidence of the need to follow OSA patients with dysphagia, for which the infective risk is certainly greater. 


\section{Conclusions}

Our data demonstrate that all OSA patients should undergo a complete ENT exam before starting CPAP therapy, which includes assessment of swallowing. The importance of this recommendation lies in both the choice of the CPAP mask (orofacial $v s$ nasal) and in the need to predict the need of high CPAP pressure setting to resolve apnoea episodes in the presence of dysphagia with the aim of obtaining satisfactory rates of adherence and tolerance to therapy. Indeed, it is acknowledged that pressures that are too high are associated with compliance issues in long-term CPAP therapy. However, the age of patients belonging to the dysphagic group was higher compared with the non-dysphagic group, and therefore age acts as a confounding factor in predicting the use of higher CPAP titration pressures. Our data also highlight that it is possible to identify a "new category" of OSA patients, "dysphagic OSA", who need strict follow-up considering that they need higher levels of positive airway pressure in PAP therapy and they might present a higher risk of developing pneumonia compared with non-dysphagic OSA patients. Finally, our study is in line with the recent literature in otolaryngology in confirming the opportunity to consider OSA as a complex pathology that requires a multidisciplinary approach for diagnosis and follow-up, in order to provide personalised and effective long-term treatment.

\section{Conflict of interest statement}

The authors declare no conflict of interest.

\section{Funding}

The authors received the "Research Projects of National Relevance 2017 n. 2017JNKCYZ" funding for this work.

\section{Authors' contributions}

Conceptualization: LM, GC, GC, MLF, OR; Data curation: LM, GC, VDL, GC, MLF; Formal analysis: LM, GC, VDL; Writing - original draft: LM, GC, VDL, MLF; Writing review and editing: LM, GC, VDL, GC, CS, VDN, NAAQ, GEC, OR, MLF.

\section{Ethical consideration}

The study was approved by the Institutional Review Board of teaching hospital Policlinico of Bari (protocol number 6750).

The research was conducted ethically, with all study procedures being performed in accordance with the requirements of the World Medical Association's Declaration of Helsinki.

Written informed consent was obtained from each patient for study participation and data publication.

\section{References}

1 Strollo PJ Jr, Rogers RM. Obstructive sleep apnea. N Engl J Med 1996;334:99-104. https://doi.org/10.1056/NEJM199601113340207

2 Peppard PE, Young T, Palta M, et al. Prospective study of the association between sleep-disordered breathing and hypertension. $N$ Engl J Med 2000;342:1378-1384. https://doi.org/10.1056/ NEJM200005113421901

3 Lindberg E, Carter N, Gislason T, et al. Role of snoring and daytime sleepiness in occupational accidents. Am J Respir Crit Care Med 2001;164:2031-2035. https://doi.org/10.1164/ajrccm.164.11.2102028

4 Berry RB, Budhiraja R, Gottlieb DJ, et al. American Academy of Sleep Medicine. Rules for scoring respiratory events in sleep: update of the 2007 AASM Manual for the Scoring of Sleep and Associated Events. Deliberations of the Sleep Apnea Definitions Task Force of the American Academy of Sleep Medicine. J Clin Sleep Med 2012;8:597-619. https://doi.org/10.5664/jcsm.2172

5 Ruehland WR, Rochford PD, O'Donoghue FJ, et al. The new AASM criteria for scoring hypopneas: impact on the apnea hypopnea index. Sleep 2009;32:150-157. https://doi.org/10.1093/sleep/32.2.15

6 Weaver TE, Maislin G, Dinges DF, et al. Relationship between hours of CPAP use and achieving normal levels of sleepiness and daily functioning. Sleep 2007;30:711-719. https://doi.org/10.1093/ sleep/30.6.711

7 Dempsey JA, Skatrud JB, Jacques AJ, et al. Anatomic determinants of sleep-disordered breathing across the spectrum of clinical and nonclinical male subjects. Chest 2002;122:840-851. https://doi. org/10.1378/chest.122.3.840

8 Cahali MB, Soares CF, Dantas DA, et al. Tonsil volume, tonsil grade and obstructive sleep apnea: is there any meaningful correlation? Clinics (Sao Paulo) 2011;66:1347-1352. https://doi.org/10.1590/ s1807-59322011000800007

9 Zonato AI, Bittencourt LR, Martinho FL, et al. Association of systematic head and neck physical examination with severity of obstructive sleep apnea-hypopnea syndrome. Laryngoscope 2003;113:973-980. https://doi.org/10.1097/00005537-200306000-00011

10 Ayas NT, Drager LF, Morrell MJ, et al. Update in sleep-disordered breathing 2016. Am J Respir Crit Care Med 2017;195:1561-1566. https://doi.org/10.1164/rccm.201701-0048UP

11 Teramoto S, Ishii T, Matsuse T. Relationship between swallowing function and gas exchange during day and night in patients with obstructive sleep apnea syndrome. Dysphagia 2001;16:249-253. https:// doi.org/10.1007/s00455-001-0083-7

12 Friedman M, Salapatas AM, Bonzelaar LB. Updated Friedman staging system for obstructive sleep apnea. Adv Otorhinolaryngol 2017;80:41-48. https://doi.org/10.1159/000470859

13 Vicini C, De Vito A, Benazzo M, et al. The nose oropharynx hypopharynx and larynx (NOHL) classification: a new system of diagnostic standardized examination for OSAHS patients. Eur Arch Otorhinolaryngol 2012;269:1297-300. https://doi.org/10.1007/ s00405-012-1965-Z

14 Neubauer PD, Rademaker AW, Leder SB. The Yale Pharyngeal Residue Severity Rating Scale: an anatomically defined and imagebased tool. dysphagia. 2015;30:521-528. https://doi.org/10.1007/ s00455-015-9631-4

15 Ginocchio D, Alfonsi E, Mozzanica F, et al. Cross-cultural adaptation and validation of the italian version of SWAL-QOL. Dysphagia 2016;31:626-634. https://doi.org/10.1007/s00455-016-9720-Z

16 Bhutada AM, Broughton WA, Focht Garand KL. Obstructive sleep apnea syndrome (OSAS) and swallowing function - a systematic review. Sleep Breath 2020;24:791-799. https://doi.org/10.1007/ s11325-020-02037-w 
17 Schindler A, Mozzanica F, Sonzini G, et al. Oropharyngeal dysphagia in patients with obstructive sleep apnea syndrome. Dysphagia 2014;29:44-51. https://doi.org/10.1007/s00455-013-9474-9

18 Jäghagen EL, Berggren D, Isberg A. Swallowing dysfunction related to snoring: a videoradiographic study. Acta Otolaryngol 2000;120:438443. https://doi.org/10.1080/000164800750000702

19 Valbuza JS, de Oliveira MM, Zancanella E, et al. Swallowing dysfunction related to obstructive sleep apnea: a nasal fibroscopy pilot study. Sleep Breath 2011;15:209-213. https://doi.org/10.1007/ s11325-010-0474-9

20 Valarelli LP, Corradi AMB, Grechi TH, et al. Cephalometric, muscular and swallowing changes in patients with OSAS. J Oral Rehabil 2018;45:692-701. https://doi.org/10.1111/joor.12666

21 Eckert DJ, White DP, Jordan AS, et al. Defining phenotypic causes of obstructive sleep apnea. Identification of novel therapeutic tar- gets. Am J Respir Crit Care Med 2013;188:996-1004. https://doi. org/10.1164/rccm.201303-0448OC

22 Caparroz FA, de Almeida Torres Campanholo M, Sguillar DA, et al. A pilot study on the efficacy of continuous positive airway pressure on the manifestations of dysphagia in patients with obstructive sleep apnea. Dysphagia 2019;34:333-340. https://doi.org/10.1007/ s00455-018-9944-1

23 Cabre M, Serra-Prat M, Palomera E, et al. Prevalence and prognostic implications of dysphagia in elderly patients with pneumonia. Age Ageing 2010;39:39-45. https://doi.org/10.1093/ageing/afp100

24 Su VY, Liu CJ, Wang HK, et al. Sleep apnea and risk of pneumonia: a nationwide population-based study. CMAJ 2014;186:415-21. https:// doi.org/10.1503/cmaj.131547

25 Chiner E, Llombart M, Valls J, et al. Association between obstructive sleep apnea and community-acquired pneumonia. PLoS One 2016;11:e0152749. https://doi.org/10.1371/journal.pone.0152749 